\title{
Descomposición de hojarasca de las especies leñosas nativas y exóticas más abundantes del pedemonte de las Yungas, Tucumán, Argentina
}

\author{
Romina D. FernándeZ ${ }^{\bowtie}$ \& Roxana Aragón \\ CONICET, Instituto de Ecología Regional, Facultad de Ciencias Naturales e IML. Universidad Nacional de Tucumán, Argentina.
}

\begin{abstract}
Resumen. Las especies exóticas pueden impactar en el proceso de descomposición, ya sea de manera directa (diferencias en calidad y /o cantidad de hojarasca) o de manera indirecta (modificando las condiciones micro ambientales y la abundancia y/o actividad de descomponedores). Los objetivos de este trabajo fueron: I) comparar la descomponibilidad de las especies exóticas y nativas más abundantes de los bosques secundarios del pedemonte de la Sierra de San Javier, Tucumán, II) analizar el efecto de las condiciones micro ambientales sobre la tasa de descomposición de un sustrato común y III) evaluar el efecto del tamaño de malla de las bolsas de descomposición en la descomposición de hojarasca. Para ello incubamos hojarasca de 7 especies nativas y 7 exóticas bajo condiciones estándares durante un año y hojarasca de Populus deltoides (sustrato común) en parches de bosques secundarios nativos e invadidos por Ligustrum lucidum en bolsas con dos tamaños de malla. La pérdida de peso no fue diferente entre el conjunto de especies nativas y exóticas pero si se encontraron diferencias interespecíficas. La constante de descomposición del sustrato común fue menor en los bosques invadidos por lo que L. lucidum modificaría la composición y/o actividad de organismos descomponedores y generaría cambios en las condiciones micro ambientales que determinan la descomposición. Por otra parte, la descomposición fue mayor en bolsas con malla grande, lo cual podría deberse al acceso de macro invertebrados. Nuestros resultados sugieren la ausencia de un patrón de descomposición en el conjunto de especies nativas y exóticas y la potencial influencia que tendría L. lucidum en los factores que controlan la descomposición.
\end{abstract}

[Palabras clave: plantas exóticas, Ligustrum lucidum, bolsas de descomposición, constante de descomposición, Yungas]

\begin{abstract}
Aвstract. Leaf litter decomposition of the most abundant native and exotic woody species in the piedmont of Yungas forest, Tucumán, Argentina: Exotic species may influence the process of decomposition, either directly (through differences in the quality and/or quantity of leaf-litter) or indirectly (by altering microclimatic conditions and the abundance and/or activity of decomposers). The aims of our study were: I) to compare the decomposability of the most abundant exotic and native species in secondary forests of the Sierra de San Javier, Tucumán, II) to assess the effect of microenvironemtal conditions on the rate of decomposition of an homogeneous substrate and III) to evaluate the effect of the litterbags mesh size on the decomposition process. To do this, we incubated leaf-litter of 7 native and 7 exotic species under standard conditions for one year and also leaf-litter of Populus deltoides (homogeneous substrate) in native and invaded by Ligustrum lucidum secondary forest patches in bags with two mesh sizes. Native and exotic species did not differ in their weight loss rates, but there were interspecific differences. The decay constant of the homogeneous substrate was lower in invaded patches so it is possible that L. lucidum modify the composition and /or activity of decomposing organisms and generate changes in micro-environmental conditions. In addition, decomposition was higher in bags with larger mesh size. This pattern may be explained by the differential access of macro invertebrates. Our results suggest the absence of a pattern of decay in native and exotic species as groups, and the potential influence that L. lucidum would have over the factors controlling littler decomposition.
\end{abstract}

[Keywords: exotic plants, Ligustrum lucidum, litterbags, litter decay constant, Yungas]

\section{INTRODUCCIÓN}

La invasión de especies exóticas puede afectar la estructura de una comunidad (e.g., diversidad, riqueza, cobertura), su dinámica (e.g., mortalidad, tasa de reclutamiento) y también diversos procesos ecosistémicos (e.g., tasa de productividad, descomposición y ciclado de nutrientes) (Vitousek et al. 1997; Mack et al. 2000; Kolar \& Lodge, 2001; Ashton et al. 2005). La descomposición de la materia orgánica juega un papel central en

$\triangle$ romina_d_fernandez@yahoo.com el funcionamiento y la productividad de los ecosistemas terrestres ya que determina el ciclado de nutrientes y su disponibilidad para las plantas y la biota edáfica (Meentemeyer 1978; Melillo et al. 1982; Swift \& Anderson 1989; Aerts1997). La descomposición puede ser afectada por la invasión de especies exóticas que puede generar cambios en la cantidad y/o calidad de hojarasca, en las condiciones ambientales (e.g., humedad del suelo, disponibilidad de luz) y en la comunidad de descomponedores (e.g., cambios en la riqueza 
o en la abundancia de distintos grupos de descomponedores) (Meentemeyer 1978; Melillo et al. 1982; Cornelissen 1996; Wardle \& Lavelle 1997; Cornwell et al. 2008).

Diversos estudios identificaron una serie de rasgos que caracterizan a las especies invasoras exitosas. Entre ellas son comunes las especies con características de pioneras (Funk \& Vitousek 2007). Por ejemplo, Baruch \& Goldstein (1999) encontraron que las invasoras poseen elevada área foliar específica, alta tasa de fotosíntesis, y bajos costos de construcción de hojas. Debido a estas características se esperaría que las invasoras presenten una mayor tasa de descomposición (Ehrenfeld 2003; Tecco et al. 2010). Sin embargo, en muchos ecosistemas presentan un amplio rango de atributos y diferentes estrategias de colonización por lo que su tasa de descomposición y sus potenciales efectos sobre el ecosistema podrían depender de la especie (Furey et al. 2014).

En los bosques secundarios de la Sierra de San Javier (Tucumán, Argentina) se registraron 15 especies arbóreas exóticas que tienen su mayor abundancia en el pedemonte y en la selva montana del Este de la Sierra (Grau \& Aragón 2000). Aunque todas estas especies son leñosas presentan características que las diferencian tales como su porte (arbustos o árboles), su tolerancia a la sombra (pioneras o tardías) y su fenología foliar (caducas o perennes). En muchos casos se ha encontrado que estas características se relacionan con la tasa de descomposición de hojarasca (Cornelissen 1996; Pérez-Harguindeguy et al. 2000; Tecco et al. 2010). Además, el impacto de una especie en el ecosistema está relacionado no solo con su tasa de descomposición en sí sino también con su abundancia (Furey et al. 2014).

Una de las exóticas más abundantes en la Sierra de San Javier es Ligustrum lucidum W.T. Aiton (Oleaceae), que en los últimos 20 años mostró una notable expansión en las Yungas (de 100 a 500 ha aproximadamente, Montti com. pers.) y que presenta un potencial para invadir áreas naturales aún en ausencia de disturbios antrópicos (Gurvich et al. 2005; Tecco et al. 2006). Esta especie arbórea es tolerante a la sombra, posee alta capacidad de rebrote, es perennifolia y forma parches casi monoespecíficos (Aragón \& Groom 2003; Lichstein et al. 2004). La tasa de descomposición de la hojarasca en estos parches monoespecíficos sombríos y con menor diversidad de especies arbóreas podría variar frente a cambios en los tres factores que influyen en la descomposición: la calidad de la hojarasca, las condiciones ambientales y la comunidad de descomponedores (Swift \& Anderson 1989; Aerts 1997).

Los objetivos de este trabajo fueron (1) evaluar la descomponibilidad de la hojarasca de 7 especies nativas y 7 especies exóticas abundantes en el pedemonte de la Sierra de San Javier; (2) analizar el efecto de las condiciones micro ambientales sobre la tasa de descomposición de un sustrato común (hojas de Populus deltoides W. Bartram ex Marshall) incubado en parches de bosques nativos y bosques invadidos por L. lucidum y; (3) evaluar el efecto del tamaño de la malla de las bolsas de descomposición sobre la tasa de descomposición de hojarasca de un sustrato común en ambos tipos de bosques. El tamaño de malla es un indicador de la accesibilidad de los distintos grupos de descomponedores (micro, meso y macrofauna) cuya importancia relativa puede ser afectada por la invasión de especies exóticas. Las hipótesis planteadas establecen que (1) Las especies exóticas presentan alta tasa de crecimiento y capacidad fotosintética (Peñuelas et al. 2010) que en general se asocia con elevada concentración de nutrientes en las hojas que permite una descomposición rápida de su hojarasca, por lo que se espera que la descomposición sea mayor en el conjunto de especies exóticas; (2 y 3) Los bosques de ligustro, más densos y sombríos, modifican la humedad y la temperatura del suelo lo cual influiría directamente en la abundancia y actividad de organismos descomponedores. Por esto se espera que la tasa de descomposición de hojarasca incubada en parches de bosques invadidos sea menor que la tasa de descomposición de hojarasca en parches de bosques nativos.

\section{MÉTOdos}

\section{Área de estudio y diseño experimental}

El estudio se realizó en la localidad de Horco Molle, en el pedemonte de la Sierra de San Javier $\left(26^{\circ} 70^{\prime} \mathrm{S}, 6^{\circ} 35^{\prime} \mathrm{O}\right)$, Tucumán, Argentina. El área forma parte del Parque Biológico Sierra de San Javier, área protegida que depende de la Universidad Nacional de Tucumán. La vegetación de la zona corresponde al piso montano inferior de la Provincia Fitogeográfica de las Yungas (Cabrera 1976). Los suelos pertenecen al orden de los Entisoles (Sesma et al. 2010), la temperatura media anual es de $18^{\circ} \mathrm{C}$ y el rango promedio de precipitaciones va desde 900 hasta $1448 \mathrm{~mm}$ ocurriendo la mayoría durante el verano (Hunzinger 1997). Se trabajó 
usando un diseño pareado con 5 parches de bosques secundarios dominados por especies nativas y 5 parches dominados por L. lucidum. Los parches apareados distaban no más de $500 \mathrm{~m}$ entre sí y presentaban condiciones similares de pendiente, edad y características del suelo (Ayup et al. 2014). Solo diferían en la abundancia relativa de L. lucidum que era mayor en parches invadidos, y en la disponibilidad de luz y humedad edáfica que eran mayores en parches nativos (Ayup et al. 2014). Para más detalles sobre el área de estudio ver Aragón et al. (2014). Los parches fueron considerados réplicas para el experimento a campo $(n=5)$.

\section{Selección de especies y recolección de hojarasca}

Se seleccionaron 7 especies nativas y 7 especies exóticas abundantes en los bosques secundarios nativos y exóticos (invadidos por L. lucidum) de HorcoMolle(Tabla 1). Además se recolectóhojarasca de $P$. deltoides de plantaciones cercanas al área de trabajo. Se eligió este género como sustrato común para incubar en parches de ambos tipos de bosques porque ya fue utilizado en estudios similares (Vivanco \& Austin 2008) y al no estar presente en los sitios de estudio, su descomposición resultante brindará información sobre la descomposición del sistema, independientemente de la calidad del sustrato (Orwin et al. 2006; Vaieretti et al. 2010). El material vegetal se recolectó mediante trampas de hojarasca, durante abril y agosto de 2011, construidas con bolsas de plástico y colocadas a una altura aproximada de $80 \mathrm{~cm}$ del suelo bajo las especies seleccionadas. El material vegetal, que consistía en hojas senescentes y sin signos de ataque de herbívoros o patógenos de al menos cinco individuos maduros de cada especie, se retiró cada 7 días de las trampas y se conservó en bolsas de papel para su posterior procesamiento.

\section{Preparación de la hojarasca}

El material de cada especie se clasificó y se secó al aire. Se pesaron muestras de $2 \mathrm{~g}$ de hojarasca y se colocaron en bolsas de fibra de vidrio (bolsas de descomposición) de $20 \times 20 \mathrm{~cm}$, con un tamaño de malla de $2 \mathrm{~mm}$ ( 12 bolsas $\times 14$ especies $=168$ bolsas $)$. De la misma manera se prepararon muestras de 2 g de hojarasca del sustrato común (P. deltoides) y se colocaron en bolsas de $2 \mathrm{~mm}$ de malla (40 bolsas) y en bolsas de $20 \times 20 \mathrm{~cm}$ con un tamaño de malla de $5 \mathrm{~mm}$ (40 bolsas), estas últimas permiten el acceso de macro invertebrados. Adicionalmente, para cada especie se tomaron tres sub-muestras de $2 \mathrm{~g}$ de hojas secadas al aire para calcular el contenido de humedad. Debido a que se detectó que la hojarasca conservaba excedente de suelo luego de limpiarla con pincel, se corrigió por cenizas (Harmon \& Lajtha 1999; Vivanco \& Austin 2006; Furey 2010). Para ello se tomaron tres submuestras de cada especie y se pesaron. Dichas submuestras se colocaron en una mufla a $500^{\circ} \mathrm{C}$ durante 4 horas y luego se pesaron sus cenizas. De este modo, se calculó el peso seco remante verdadero o remanente de materia orgánica (MO) utilizado como variable para los análisis.

\section{Incubación de la hojarasca}

Descomponibilidad de hojarasca de especies nativas y exóticas: En septiembre de 2011 se colocaron simultáneamente las 168 bolsas con hojarasca de las especies nativas y exóticas en una cama de descomposición construida en una porción de terreno del invernáculo del Instituto de Ecología Regional (IER) en Horco Molle. Para la instalación del experimento se acondicionó un área de aproximadamente $5 \times 4 \mathrm{~m}$. Allí previamente a la incubación de las bolsas se removió la hojarasca y la vegetación existente. Las muestras se colocaron

Tabla 1. Familias, origen, fenología foliar y tipo de reclutamiento de las especies nativas y exóticas consideradas en este estudio. Los nombres científicos corresponden a los lineamientos establecidos en el Catálogo de las Plantas Vasculares del Conosur (http:/ / www2.darwin.edu.ar/Proyectos/FloraArgentina/FA.asp). *Tipo de reclutamiento según Grau \& Aragón 2000.

Table 1. Family, status, leaf phenology and type of recruitment of native and exotic species considered in this study.

\begin{tabular}{lcccc}
\hline Especie & Família & Estatus & $\begin{array}{c}\text { Fenología } \\
\text { foliar }\end{array}$ & $\begin{array}{c}\text { * Tipo de } \\
\text { reclutamiento }\end{array}$ \\
\hline Allophylus edulis (A. St. Hil., A. Juss. \& Cambess.) Hieron. ex Niederl. & Sapindaceae & Nativa & perenne & Tolerante \\
Blepharocalyx salicifolius (Kunth) O. Berg & Myrtaceae & Nativa & perenne & Tolerante \\
Cinnamomun porphyrium (Griseb.) Kosterm. & Lauraceae & Nativa & perenne & Tolerante \\
Cupania vernalis Cambess. & Sapindaceae & Nativa & perenne & Tolerante \\
Myrsine laetevirens (Mez) Arechav. & Myrsinaceae & Nativa & perenne & Tolerante \\
Tecoma stans (L.) Juss. ex Kunth & Bignoniaceae & Nativa & perenne & Pionera \\
Tipuana tipu (Benth.) Kuntze & Fabaceae & Nativa & perenne Pionera longeva \\
Citrus aurantium L. & Rutaceae & Exótica & perenne & tolerante \\
Eriobotrya japonica (Thunb.) Lindl. & Rosaceae & Exótica & perenne & pionera \\
Ligustrum lucidum W.T. Aiton & Oleaceae & Exótica & perenne Pionera rebrote \\
Ligustrum sinense Lour. & Oleaceae & Exótica & perenne Pionera rebrote \\
Morus alba L. & Moraceae & Exótica & caduca & Pionera \\
Persea americana Mill. & Lauraceae & Exótica & Perenne & Pionera \\
Psidium guajava L. & Myrtaceae & Exótica & perenne & Pionera \\
\hline
\end{tabular}


al azar y se cubrieron con parte de los restos vegetales extraídos durante la limpieza con el fin de homogeneizar las condiciones físicas de tal manera que, las diferencias entre los valores de descomponibilidad obtenidos se deban a la calidad de la hojarasca (Cornelissen 1996, 1999). El experimento se mantuvo hasta septiembre de 2012. En cada una de las recolecciones realizadas después de los 95, 140, 246 y 365 días de incubación se extrajeron 3 bolsas de cada especie $(n=3)$. Las muestras obtenidas en cada recolección se limpiaron con pincel para eliminar restos de suelo y material vegetal extraño a las muestras, se secaron en estufa a $60^{\circ} \mathrm{C}$ durante 3 días hasta obtener peso constante y se pesaron.

Efecto de las condiciones micro ambientales y del tamaño de malla en la descomposición: El efecto de las condiciones micro ambientales que generan ambos tipos de bosques sobre la tasa de descomposición de hojarasca se evaluó mediante la incubación, a principios de agosto de 2011, de las bolsas de $2 \mathrm{~mm}$ de malla con hojarasca de $P$. deltoides, en 5 parches de bosques secundarios dominados por especies nativas, y 5 parches de bosques dominados por $L$. lucidum. En cada uno de los parches se seleccionó al azar una parcela de $2 \times 2 \mathrm{~m}$ donde se colocaron las bolsas una al lado de la otra de manera similar a la descripta para el experimento anterior. En las mismas parcelas se colocaron simultáneamente las bolsas de malla más grande $(5 \mathrm{~mm})$ para evaluar el efecto del tamaño de la malla. En cada una de las recolecciones, realizadas a $\operatorname{los} 48,120,264$ y 365 días de incubación, se retiró 1 bolsa de malla chica y 1 bolsa de malla grande de cada uno de los parches nativos y exóticos $(n=5)$. Las muestras recolectadas se procesaron en el laboratorio de la manera descripta anteriormente.

Las tasas de descomposición fueron estimadas a partir de la pérdida de peso seco para lo cual se utilizó un modelo de decaimiento exponencial $k=-\operatorname{Ln}(\mathrm{Mt} / \mathrm{M} 0) / t$, donde $k$ es la constante de descomposición, $\mathrm{M}_{0}$ y $\mathrm{M}_{\mathrm{t}}$ corresponden al peso seco remanente inicial y final después del periodo de estudio, y t es el tiempo (año) (Swift \& Anderson 1989; Perez-Harguindeguy et al. 2013).

\section{Análisis de datos}

Exploramos la existencia de diferencias de descomponibilidad de hojarasca entre las especies nativas y exóticas mediante un análisis de varianza (ANOVA) utilizando la proporción de peso seco remanente de la última recolección como variable de respuesta, al origen (exótica o nativa) como variable categórica (efecto fijo) y a las especies (efecto aleatorio) como anidadas dentro de origen. Se usó la función lme del paquete nlme de R ( $R$ Development Core Team, 2010). Para evaluar la descomposición del sustrato común (P. deltoides) en los dos tipos de bosques, se realizó un ANOVA con la constante de descomposición $(k)$ como variable de respuesta y considerando los efectos de tipo de bosque, tipo de bolsa (malla chica y grande) y su interacción como fuentes de variación y un diseño en bloques (5 pares de sitios). Los valores de $k$ se transformaron con logaritmo considerando el supuesto de homogeneidad de varianzas. Las medias de $k$ se compararon con LSD-Fisher como test a posteriori. Estos análisis se realizaron con el paquete estadístico InfoStat (Di Renzo et al. 2013).

\section{Resultados}

No se encontraron diferencias significativas entre los promedios de peso seco remanente para las especies nativas y exóticas como grupo $\left(\mathrm{F}_{1,12}\right.$ para origen $\left.=0.44 ; \mathrm{p}=0.51\right)$ pero sí se observaron diferencias interespecíficas. La diferencia entre las medias de peso remanente de estos dos grupos fue del $8 \%$, mientras que las diferencias entre las especies que presentaron la menor y mayor

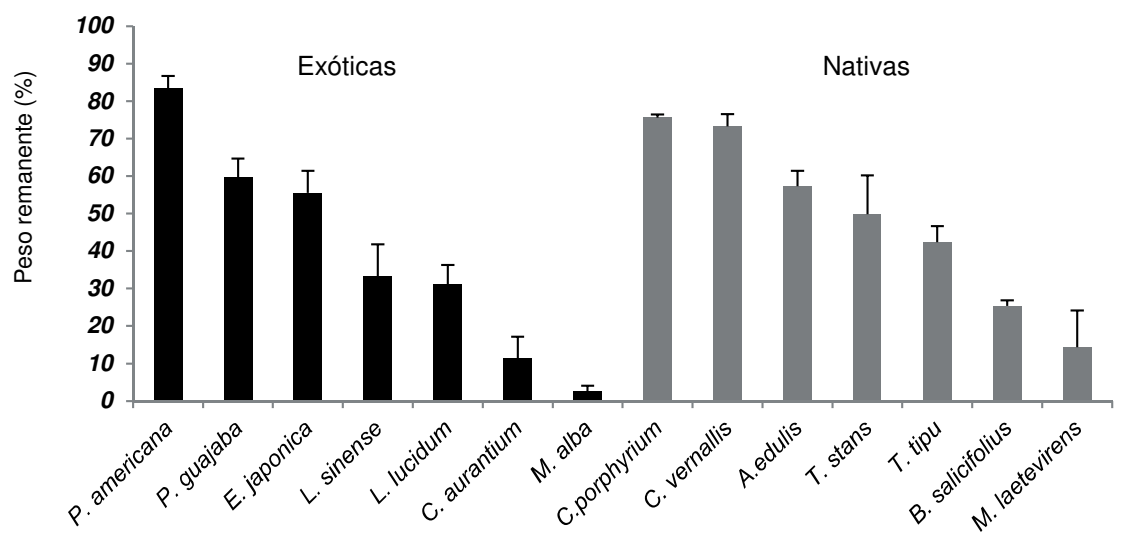

Figura 1. Proporción de peso seco remanente de las especies nativas y exóticas después de 365 días de incubación en la cama de descomposición. Las barras representan las medias $(n=3)$ con su error estándar. Los nombres completos de las especies se encuentran en Tabla 1.

Figure 1. Proportion of remaining dry weight of native and exotic species after 365 days of incubation in the bed of decomposition. Bars represent the means $(n=3)$ with standard errors. The full scientific names of the species are included in Table 1. 
descomposición dentro de cada grupo fue del 62 y del $80 \%$ para nativas y exóticas respectivamente. Es decir, la variabilidad asociada a las diferencias entre especies fue mayor a la asociada a la diferencia entre exóticas y nativas en promedio. Las especies que presentaron mayor peso seco remanente (i.e., menor descomposición) después de un año de incubación fueron Persea americana, Psidium guajava (exóticas), Cinnamomun porphyrium y Cupania vernalis (nativas). Las especies con mayor descomposición fueron Morus alba, Citrus aurantium (exóticas) y Myrsine laetevirens y Blepharocalyx salicifolius (nativas) (Figura 1).

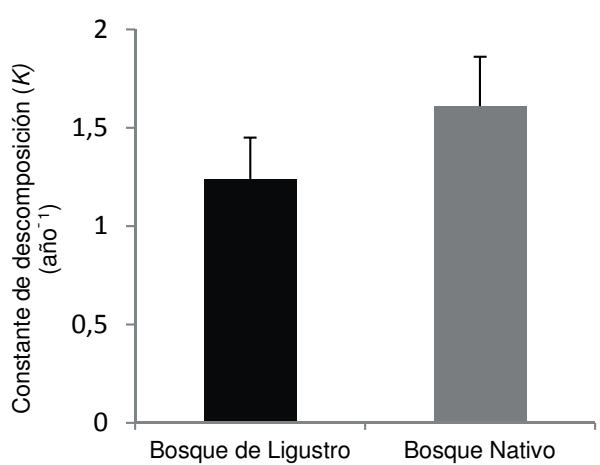

Figura 2. Constante de descomposición $(k)$ de la hojarasca de $P$. deltoides en los dos tipos de bosques secundarios. Las barras representan la media de cada bosque $(n=5)$ con su error estándar. $\left(\mathrm{F}_{1,12}=4.47 ; \mathrm{p}=0.05\right)$.

Figure 2. Decomposition constant $(k)$ of $P$. deltoides leaflitter in the different forest types. Bars represent the means with standard errors $\left(\mathrm{F}_{1,12}=; 4.47 ; \mathrm{p}=0.05\right)$.

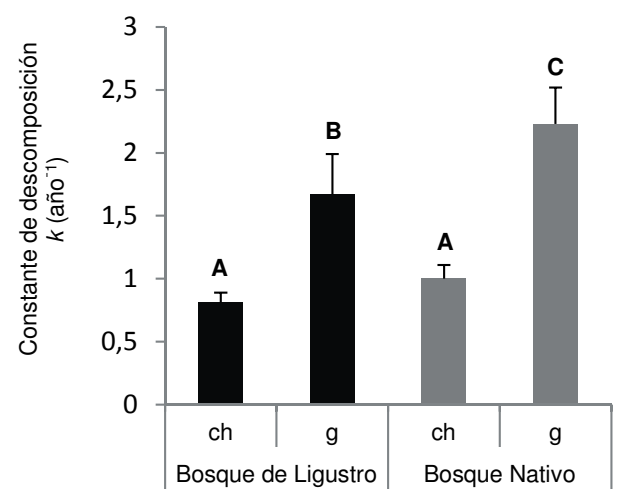

Figura 3. Constante de descomposición $(k)$ de la hojarasca de $P$. deltoides en bolsas con malla chica $2 \mathrm{~mm}(\mathrm{ch})$ y grande $5 \mathrm{~mm}(\mathrm{~g})$ incubadas en ambos tipos de bosques secundarios. Las barras representan las medias de cada tipo de bosque $(n=5)$ con sus errores estándares. Diferentes letras indican diferencias significativas $(\mathrm{p}<0.05)$.

Figure 3. Decomposition constant $(k)$ of $P$. deltoides leaflitter incubated in small $2 \mathrm{~mm}$ (ch) or large $5 \mathrm{~mm}(\mathrm{~g})$ mesh bags in both types of secondary forests. Bars represent the means $(n=5)$ with standard errors. Different letters indicate significant differences $(p<0.05)$.
La hojarasca de $P$. deltoides, utilizado como sustrato común para evaluar las diferencias debidas a la variación microambiental, presentó una mayor descomposición en los parches de bosques nativos $\left(\mathrm{F}_{1,12}=4.47\right.$; $\mathrm{p}=$ 0.05) (Figura 2). Además, este mísmo sustrato incubado en bolsas con distinto tamaño de malla mostró mayor descomposición en las bolsas de malla grande $(5 \mathrm{~mm})\left(\mathrm{F}_{1,12}=31.40\right.$; $\mathrm{p}=0.0001)$ (Figura 3). La diferencia entre las bolsas de distinto tamaño de malla fue similar en ambos tipos de bosques $\left(\mathrm{F}_{1,12}\right.$ para tipo de bolsa*tipo de bosque $=0.28 ; \mathrm{p}=0.60$ ).

\section{DISCUSIÓN}

Contrario a nuestras predicciones, la descomposición de la hojarasca del conjunto de especies exóticas fue similar a la del conjunto de especies nativas después de un año de incubación en un ambiente común. Los resultados existentes sobre descomposición de hojarasca son muy diversos. Según un metaanálisis realizado por Liao et al. (2008) los ecosistemas invadidos tienen, en general, tasas de descomposición que aproximadamente duplican las de los ecosistemas nativos. De manera similar, en una revisión realizada anteriormente se encontró que en la mayoría de los casos la hojarasca de las especies exóticas se descompone más rápido que la hojarasca de las especies nativas con las que co-ocurren (Ehrenfeld 2003). No obstante, otros estudios realizados en diferentes ecosistemas de América del Norte y mediterráneos de España evidenciaron resultadoscontrarios (Drenovsky \& Batten 2007; Godoy et al. 2010).

En nuestro estudio el rango de descomposición después un año de incubación varió entre un 14 a 76 \% en las especies nativas y entre un 3 a $83 \%$ en las especies exóticas, por lo que no se detectó un patrón que englobe a estos dos grupos, es decir que las diferencias resultaron especie-específicas. Con respecto a la descomposición de especies particulares, nuestros resultados concuerdan con los de otros trabajos locales. En el bosque serrano de Córdoba, Furey et al. (2014) encontraron que la pérdida de peso después de 43 días de incubación fue de $15 \%$ y $30 \%$ para L. lucidum y $M$. alba respectivamente, valores similares a los obtenidos en nuestro estudio. Por otro lado, para las Yungas de Salta, Gurvich et al. (2003) encontraron que la pérdida de peso de C. porphyrium a los 105 días de incubación fue de $14 \%$ mientras que en nuestro estudio a los 95 días de incubación, C. porphyrium perdió el $17 \%$ de su peso inicial. Además, la pérdida de 
peso para L. lucidum, M. alba, C. porphyrium y C. vernalis de nuestro estudio fue similar a la encontrado para estas especies en un experimento de campo realizado previamente en esta misma área (Aragón et al. 2014), por lo que nuestros valores están dentro del rango citado para estas especies.

La variación en la descomposición de las distintas especies podría deberse al amplio espectro de características foliares (e.g., área foliar específica, dureza, contenido de lignina, nitrógeno, etc.) (Asthon et al. 2005; Hobbie et al. 2006; Vivanco \& Austin 2006; Godoy et al. 2010; Peñuelas et al. 2010) en las que no se evidencia una clara distinción entre nativas y exóticas. La mayor descomposición de M. alba podría relacionarse, en parte, a su fenología foliar caduca frente a la fenología foliar perenne del resto de las especies. En general, las especies con hojas caducas presentan mayor área foliar específica lo cual se relaciona con una mayor descomposición (Cornelissen et al. 1999). Este es el caso de M. alba, que es una de las especies con mayor área foliar en estos bosques (Easdale et al. 2007; Aragón et al. 2014). Sin embargo, es importante destacar que el efecto de una especie a nivel de ecosistema depende de sus características (e.g., descomponibilidad) y de su abundancia relativa. Es por eso que si bien L. lucidum no se ubica en un extremo de descomponibilidad (su descomponibilidad no es particularmente alta ni baja) podemos predecir que su efecto a nivel de ecosistema será mayor dada su abundancia (Aragón et al. 2014; Furey et al. 2014).

Como esperábamos, debido a las características de los parches dominados por L. lucidum, la tasa de descomposición de hojarasca de $P$. deltoides fue inferior cuando se incubó en parches de bosques invadidos en comparación con la tasa de descomposición del sustrato común en parches de bosques nativos. Los sitios nativos y exóticos que forman parte de este trabajo presentan similares características del suelo tales como el $\mathrm{pH}$, el contenido de nutrientes (Ayup et al. 2014) y temperatura del suelo y difirieren en el contenido de humedad del suelo y en la disponibilidad de luz, que es menor en los sitios invadidos (Ayup et al. 2014). Las condiciones ambientales influyen en la composición y actividad de los descomponedores y por lo tanto en la descomposición de hojarasca. Algunos invertebrados descomponedores que desempeñan un rol importante en la fragmentación de hojarasca son sensibles a las condiciones de humedad y temperatura, como es el caso de los isópodos y diplópodos (David \& Handa 2010) por lo que es probable que el mayor contenido de humedad en parches nativos acelere la descomposición en esos sitios. Experimentos previos que comparan la descomposición de hojarasca en diferentes sitios (nativos e invadidos) difieren en los resultados, donde algunas especies responden a diferencias de sitios mientras que otras no (Kourtev et al.2002). En nuestro estudio, y contrario a lo hallado por Ashton et al. (2005), encontramos que la descomposición de hojarasca del sustrato común fue mayor en parches de bosques secundarios nativos, diferencias que podrían explicarse por las diferencias de humedad y disponibilidad de luz.

Si bien en este trabajo no estudiamos específicamente a las comunidades de descomponedores, ellas constituyen un factor importante en la descomposición y su rol es determinante en algunos sistemas (Seastedt 1984; Hättenschwiler \& Gasser 2005). Por lo tanto, no es sorprendente que la hojarasca de $P$. deltoides incubada en bolsas con malla más pequeña, que excluye a algunos integrantes de la mesofauna y a la macrofauna, haya presentado una menor descomposición en ambos tipos de bosques secundarios. Estos organismos fragmentan el material vegetal lo cual facilita la lixiviación de varios compuestos y aumenta el área superficial de la hojarasca; una mayor área favorece el desarrollo de poblaciones microbianas y finalmente aceleran el proceso de descomposición (Swift \& Anderson 1989; Wall \& Moore 1999; Reynolds \& Hunter 2001). A pesar de que en ambos tipos de bosques hubo diferencias en la descomposición según el tipo de bolsa, esa diferencia fue algo menor en los ligustrales. Esto podría deberse a la presencia de diferentes comunidades de organismos descomponedores de hojarasca en ambos tipos de bosques.

En resumen, las características de la hojarasca de cada especie ejercerían un control importante en su descomposición y esas características no parecen estar relacionadas directamente a su condición de nativa o exótica. Por otro lado, L. lucidum afectaría los diferentes factores que influyen en la descomposición de hojarasca. La formación de parches prácticamente monoespecíficos de L. lucidum en el pedemonte de la Sierra de San Javier generaría condiciones micro ambientales diferentes a las generadas por especies nativas lo cual influiría en la composición, abundancia 
y actividad de descomponedores y por lo tanto afectaría a la tasa de descomposición. Por lo tanto, para conocer y predecir el efecto de las especies exóticas sobre la descomposición se requieren estudios comparativos que evalúen de manera simultánea los factores que influyen en este proceso (e.g., humedad, temperatura, abundancia de distintos grupos de descomponedores) y que consideren mezclas representativas de la disponibilidad de hojarasca de cada tipo de bosque.

Agradecimientos: Este estudio fue financiado por una beca del Consejo de Investigaciones de la Universidad Nacional de Tucumán de RF, por el proyecto 267G 471 de RA y por el PICT 1382 dirigido por R Grau. Agradecemos a M Ayup, L Montti y R Grau por sus aportes en las distintas etapas de la tesina a partir de la cual surgió este trabajo y a F Biganzoli y E Araoz por su colaboración con el análisis de datos.

\section{BIBLIOGRAFIA}

AERTS, R. 1997. Climate, leaf litter chemistry and leaf litter decomposition in terrestrial ecosystems: a triangular relationship. Oikos, 79:439-449.

Aragón, R \& M Groom. 2003. Invasion by Ligustrum lucidum (Oleaceae) in NW Argentina: early stage characteristics in different habitats types. Rev. Biol. Trop., 51:59-70.

Aragón, R; L Montti; M Ayup \& R Fernandez. 2014. Exotic species as modifiers of ecosystem processes: litter decomposition in native and invaded secondary forests of NW Argentina. Acta Oecologica, 54:21-28.

Ashton, IW; LA Hyatt; KM Howe; J Gurevitch \& MT LERDAU. 2005. Invasive species accelerate decomposition and litter nitrogen loss in a mixed deciduous forest. Ecol. Appl., 15:1263-1272.

Ayup, M; L Montti; R Aragón \& HR Grau. 2014. Invasion of Ligustrum lucidum (Oleaceae) in southern Yungas of Argentina: effects in habitat properties and avian communities. Acta Oecologica, 54:72-81.

BARUCH, Z. \& G GOLDSTEIN. 1999. Leaf construction cost, nutrient concentration, and net $\mathrm{CO}_{2}$ assimilation of native and invasive species in Hawaii. Oecologia, 121: 183-192.

CABrera, AL. 1976. Regiones Fitogeográficas de Argentina. ACME. 135 pp.

CoRnelissen, JHC. 1996. An experimental comparison of leaf decomposition rates in a wide range of temperate plant species and types. J. Ecol., 84:573-582.

Cornelissen, JHC; N Pérez-Harguindeguy; S Díaz; JP Grime; B Marzano; Et Al. 1999. Leaf structure and defence control litter decomposition rate across species and life forms in regional floras on two continents. New Phytol., 143:191-200.

Cornwell, WK; JHC Cornelissen; K Amatangelo; E DORREPAAL; V EVINER; ET AL. 2008. Plant species traits are the predominant control on litter decomposition rates within biomes worldwide. Ecol. Letters, 11:1065-1071.

DAVID, JF \& IT HANDA. 2010. The ecology of saprophagous macroarthropods (millipedes, woodlice) in the context of global change. Biol. Rev., 85:881-895.

Di Rienzo, JA; F Casanoves; MG Balzarini; L GonZalez; M TABLADA; ET AL. 2013. InfoStat versión 2013. Grupo
InfoStat, FCA, Universidad Nacional de Córdoba, Argentina. URL http://www.infostat.com.ar.

Drenovsky, RE \& KM BAtTen. 2007. Invasion by Aegilops triuncialis (Barb Goatgrass) slows carbon and nutrient cycling in a serpentine grassland. Biol. Inv., 9:107-116.

Easdale, TA; JR Healey; HR Grau \& A Malizia. 2007. Tree life histories in a montane subtropical forest: species differ independently by shade-tolerance, turnover rate and substrate preference. J. Ecol., 95:1234-1239.

EHRENFELD, JG. 2003. Effects of exotic plant invasions on soil nutrient cycling processes. Ecosystems, 6:503-523.

FunK, JL \& PM Vitousek. 2007. Resourse-use efficiency and plant invasión in low-resource systems. Nature, 446:1079-1081.

Furey, C. 2010. Patrones de descomposición en el Bosque Serrano de Córdoba: el efecto de las especies exóticas a través de los parches monoespecíficos, la calidad foliar y las mezclas de broza. Grade thesis. Escuela de Biología, Facultad de Ciencias Exactas, Físicas y Naturales e Universidad Nacional de Córdoba- Argentina. Pp 48.

Furey, C; PA Tecco; N Perez-Harguindeguy; MA Giorgis \& M Grossi. 2014. The importance of native and exotic plant identity and dominance on decomposition patterns in mountain woodlands of central Argentina. Acta Oecologica, 54:13-20.

Godoy, O; P Castro-Díez; RSP Van Logtestijn; JHC CORNELISSEN \& F VALLADARES. 2010. Leaf litter traits of invasive species slow down decomposition compared to Spanish natives: a broad phylogenetic comparison. Oecologia, 162:781-790.

Grau, HR \& R Aragón. 2000. Arboles invasores de la Sierra de San Javier, Tucumán, Argentina. Pp.5-20. En: Grau HR \& R Aragón (eds.), Ecología de árboles exóticos en las Yungas argentinas. LIEY, Universidad Nacional de Tucumán, Tucumán, Argentina.

Gurvich, DE; TA Easdale \&N Pérez-Harguindeguy. 2003. Subtropical montane tree litter decomposition: Links withsecondary forest types and species' shade tolerance. Austral Ecol., 28: 666-673.

Gurvich, DE; PA TeCco \& S Díaz. 2005. Plant invasions in undisturbed ecosystems: the triggering attribute approach. J.Veg.Sci., 16:723-728.

HARMON, ME \& K LAJTHA. 1999. Analysis of Detritus and Organic Horizons for Mineraland Organic Constituents. pp. 143-163. En: Robertson PG; DC Coleman; CS Bledsoe \& P Sollins (eds.). Standard soil methods for long-term ecological research. LTER New York. Oxford University Press.

Hättenschwiler, S \& P GASSER. 2005. Soil animals alter plant litter diversity effects on decomposition. Proc. Natl. Acad. Sci. USA, 102:1519-1524.

Hobbie, SE; PB Reich; J Oleksyn; M Ogdahl; R ZytKowiak; ET AL. 2006. Tree species effects on decomposition and floor dynamics in a common garden. Ecology, 87:2288-2297.

Hunzinger, H.1995. La precipitación horizontal: Su importancia para el bosque y a nivel de cuencas en la Sierra San Javier, Tucumán, Argentina. pp. 53-58. En: Brown A \& Grau HR (eds.). Investigación, conservación $y$ desarrollo en selvas subtropicales de montaña, LIEY, Tucumán.

Kolar, CS \& DM Lodge. 2001. Progress in invasion biology: predicting invaders. Trends Ecol.Evol., 16:199-204.

Kourtev, PS; JG Ehrenfeld \& WZ Huang. 2002. Enzyme activities during litter decomposition of two exotic and two native plant species in hardwood forests of New Jersey. Soil Biol. Biochem., 34:1207-1218. 
Liao, C; R PenG; Y Luo; X Zhou; X Wu et al. 2008. Altered ecosystem carbon and nitrogen cycles by plant invasion: a meta-analysis. New Phytol., 177:706-714.

Lichstein, JW; HR Grau \& R Aragón. 2004. Recruitment limitation in secondary forests dominated by an exotic tree. J. Veg. Sci., 15:721-728.

Mack, RN; D SimberlofF; WM LonSdale; H Evans; M Clout ET AL. 2000. Biotic invasions: causes, epidemiology, global consequences, and control. Ecol.Appl., 10:689-710.

Melillo, JM; JD Aber; PA Steudler \& JP SChimel. 1982. Nitrogen and lignin control of hardwood leaf litter decomposition dynamics. Ecology, 63:621-626.

MEENTEMEYER, V. 1978. Macroclimate and lignin control of litter decomposition rates. Ecology, 59:465-472.

ORWIN, KH; DA WARDLE \& LG GREENFIELD. 2006. Ecological consequences of carbon substrate identity and diversity in a laboratory study. Ecology, 87:580-593.

Peñullas, J; J Sardans; J Llusià; SM Owen; J Carnicer; et AL. 2010. Faster returns on 'leaf economics' and different biogeochemical niche in invasive compared with native plant species. Glob.Change Biol., 16:2171-2185.

Pérez Harguindeguy, N; S Díaz; JHC Cornelissen; F Vendramini; M Cabido; et al. 2000. Chemistry and toughness predict leaf litter decomposition rates over wide spectrum of functional types and taxa in central Argentina. Plant Soil, 218:21-30.

Pérez-Harguindeguy, N; S Díaz; E Garnier; S Lavorel; H POORTER; ET AL. 2013. New handbook for standardised measurement of plant functional traits worldwide. Aust. J. Bot., 61:167-234.

R Core Team. 2010. R: A language and environment for statistical computing. R Foundation for Statistical Computing, Vienna, Austria.

REYnolds, BC \& MD Hunter. 2001. Responses of soil respiration, soil nutrients, and litter decomposition to inputs form canopy herbivores. Soil Biol. Biochem., 33: 1641-1652.

Seastedt, TR. 1984. The role of microarthropods in decomposition and mineralization processes. Annu. Rev. Entomol., 29:25-46.

Sesma, PJ; EY Guido \& ME Puchulu. 2010. Geología de la ladera oriental de la Sierra de San Javier, soporte físico para la gestión territorial pp 19-32. En: Grau HR (ed). Ecología de una interfase natural-urbana. La Sierra de San Javier y el Gran San Miguel de Tucumán. EDUNT, Tucumán.

Swift, MJ \& JM Anderson. 1989. Decomposition pp. 547-569. En: Lieth, H, M Werger (eds.).Tropical Rain Forest Ecosystems, Elsevier, Amsterdam.

Tecco, PA. 2006. Riqueza, abundancia y características morfofuncionales de las especies nativas y exóticas establecidas bajo distintas condiciones de uso de la tierra en cinco ecosistemas del centro-oeste de Argentina. Tesis Doctoral. Escuela de Biología, Facultad de Ciencias Exactas, Físicas y Naturales e Universidad Nacional de CórdobaArgentina.

Tecco, PA; S Díaz; M Cabido \& C UrCelay. 2010. Functional traits of alien plants across contrasting climatic and landuse regimes: do aliens join the locals or try harder than them? J. Ecol., 98:17-27.

Vaieretti, MV; AM Cingolani; N Pérez Harguindeguy; DE GuRvich \& M CABIDO. 2010. Does decomposition of standard materials differ among grassland patches maintained by livestock? Austral Ecology, 35: 935-943.

VitouseK, PM; CM D'ANTONIO; LL LOOPE; M RejMANEK \& R WESTBROOKS. 1997. Introduced species: a significant component of human-caused global change. New Zealand J. Ecol., 21:1-16.

VIVANCO, L \& AT AUSTIN. 2006. Intrinsic effects of species on leaf litter and root decomposition: a comparison of temperate grasses from North and South America. Oecologia, 150:97-107.

Vivanco, L \& AT Austin. 2008. Tree species identity alters forest litter decomposition through long-term plant and soil interactions in Patagonia, Argentina. J. Ecol., 96:727-736.

WALL, D \& J MOORE. 1999. Interactions underground, soil biodiversity mutualism and ecosystems processes. Bioscience, 49:108-117.

WardLE, DA \& P LAVELle. 1997. Linkages between soil biota, plant litter quality and decomposition pp. 107-124. En: Cadisch, G. \& KE Giller (eds.). Plant Litter Quality and Decomposition. CAB International, Wallingford. 